\title{
A Theoretical Basis for the Scaling Law of Broadband Shock Noise Intensity in Supersonic Jets
}

\author{
Max Kandula \\ ASRC Aerospace, NASA Kennedy Space Center, Merritt Island, FL 32899, USA \\ Correspondence should be addressed to Max Kandula, max.kandula-1@ksc.nasa.gov \\ Received 8 September 2010; Accepted 27 January 2011 \\ Academic Editor: Lars Hakansson \\ Copyright () 2011 Max Kandula. This is an open access article distributed under the Creative Commons Attribution License, which \\ permits unrestricted use, distribution, and reproduction in any medium, provided the original work is properly cited. \\ A theoretical basis for the scaling of broadband shock noise intensity in supersonic jets was formulated considering linear shock- \\ shear wave interaction. Modeling of broadband shock noise with the aid of shock-turbulence interaction with special reference to \\ linear theories is briefly reviewed. A hypothesis has been postulated that the peak angle of incidence (closer to the critical angle) for \\ the shear wave primarily governs the generation of sound in the interaction process with the noise generation contribution from \\ off-peak incident angles being relatively unimportant. The proposed hypothesis satisfactorily explains the well-known scaling law \\ for the broadband shock-associated noise in supersonic jets.
}

\section{Introduction}

Noise from subsonic jets is mainly due to turbulent mixing, according to the theoretical model of Sir James Lighthill $[1,2]$. The turbulent mixing noise is essentially broadband. In perfectly expanded supersonic jets (nozzle exit plane pressure equals the ambient pressure), the large-scale mixing noise manifests itself primarily as Mach wave radiation $[3,4]$ caused by the supersonic convection of turbulent eddies with respect to the ambient fluid. In imperfectly expanded supersonic jets (nozzle exit pressure different from the ambient pressure) typical of jet and rocket exhausts at offdesign conditions, additional noise is generated in the form of broadband shock-associated noise (BBSN) emanating from shock-turbulence interaction [5] and screech tones [6] with the tonal (screech) amplitude shown to be occasioned by shock-acoustic wave interaction [7].

Figure 1 displays a typical narrowband farfield shock noise spectrum, indicating various noise components. Here, the quantity St denotes the Strouhal number $\left(f d_{j} / u_{j}\right), f$ the frequency, $d_{j}$ the nozzle exit diameter, $u_{j}$ the jet exit velocity, $p_{e}$ the nozzle exit pressure, $p_{0}$ the ambient pressure, $\phi$ the angle from the downstream jet axis, $M_{d}$ the nozzle design Mach number, and $M_{j}$ the fully expanded jet Mach number.
In imperfectly expanded supersonic jets, the rapid variation in the pressure across the nozzle exit is accompanied by a system of steady compression (oblique shock) and expansion waves (Figure 2). The structure of these shock cells was investigated by Emden [8], Prandtl [9], Rayleigh [10], Pack [11], and others. In general, these shock-expansion units interact with instability waves, vortices, turbulence, and other stream disturbances in the viscous shear layer that surrounds the inviscid region. The interaction of turbulence with shock waves leads to the generation of the broadband shock noise, which is of relatively high intensity and may form a significant component of the overall jet noise, depending on the flow conditions. The peak (characteristic) frequency of the broadband shock noise is intimately related to (varies inversely as) the shock-cell spacing which is roughly uniform over several shock cells [12]. A fundamental understanding of the mechanism by which turbulence interacts with a shock wave is thus requisite in the analysis of the complex phenomena of shock noise generation.

Lighthill [13] and Ribner [14, 15] originally suggested that the scattering of eddies by shocks could be a strong source of supersonic jet noise. The importance of source coherence, however, has not been recognized, so that only incoherent and randomly scattered sound waves had been 


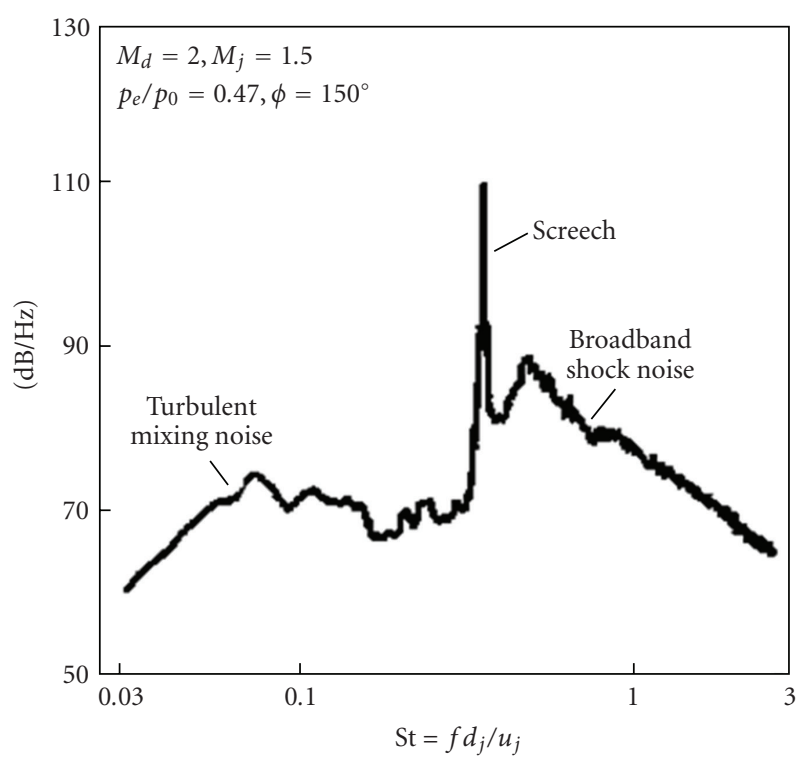

Figure 1: A typical narrowband farfield shock noise spectrum (adapted from Seiner [4]).

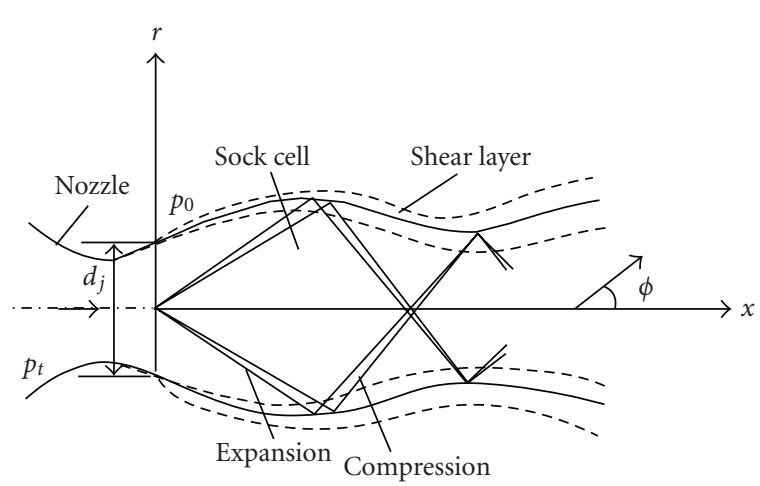

FIGURE 2: Shock cell structure in an underexpanded supersonic jet.

predicted without the peak frequency and directivity relationships. It was Harper-Bourne and Fisher [5] who first identified the detailed characteristics of BBSN with the aid of measurements from conical (convergent) nozzles and indicated the importance of source coherence. The characteristics of shock noise were also reviewed and discussed in [16]. Howe and Ffowcs Williams [17] also considered that the primary source of broadband shock-associated noise is a consequence of the interaction between large-scale structures (turbulence) and the shock structure.

Computing shock noise intensity in supersonic jets from first principles (on the basis of shock-turbulence interaction) is very difficult. The nature of the relevant noise sources is not well understood [18]. This situation is exemplified by the fact that the theories of both Lighthill [13] and Ribner [19, 20] produce shock noise intensity scaling considerably different from that indicated by the measurements.

It is the purpose of this work to investigate the scaling of broadband shock noise intensity from considerations based on linear theory for shock-vorticity interaction. It is demonstrated here that the scattering of turbulence by the leading shock wave is related to the measured shock noise intensity scaling. Flight effects are excluded from consideration here. Also screech effects are not relevant to this investigation. This work is primarily based on [21].

\section{Measurements and Characteristics of Broadband Shock Noise}

Harper-Bourne and Fisher [5] were the first to identify significant features of shock-noise in considerable detail based on their static jet measurements from conical nozzles. The intensity of BBSN is shown to be primarily a function of the nozzle (operating) pressure ratio NPR $=p_{t} / p_{0}$, where $p_{t}$ is the stagnation (reservoir) pressure. For a given radiation direction, the measured overall sound intensity $I$ has been observed to scale as

$$
I \propto \beta^{4}
$$

where

$$
\beta=\left(M_{j}^{2}-1\right)^{1 / 2},
$$

with the isentropic relation between $p_{t} / p_{0}$ and $M_{j}$ expressed by

$$
\frac{p_{t}}{p_{0}}=\left(1+\frac{\gamma-1}{2} M_{j}^{2}\right)^{\gamma /(\gamma-1)} .
$$

In the preceding relations, the quantity $M_{j}$ represents the fully expanded jet Mach number, $\gamma$ is the isentropic exponent (ratio of specific heats), and the parameter $\beta^{2}$ characterizes the pressure jump across a normal shock at approach Mach number $M_{j}$.

Figure 3 presents the data for the overall sound power level (OASPL) at $90 \mathrm{deg}$. to the jet axis, normalized to $r / d_{j}=$ 1 , are shown for two different nozzle diameters $\left(d_{j}=25 \mathrm{~mm}\right.$, and $35 \mathrm{~mm}$ ) and at a stagnation temperature $T_{t}=290 \mathrm{~K}$, with $r$ denoting the measurement location. Equation (1a) is plotted as a dashed line, and the estimated mixing noise based on extrapolation of low-speed data at $T_{t}=290 \mathrm{~K}$ is plotted as a dotted line. Harper-Bourne and Fisher [5] found that the parameter $\beta$ correlated BBSN quite well up certain values of $\beta$ (or NPR), say $0.5<\beta<1.2$. At large NPR or $\beta$, the data begins to deviate from this law because of the presence of a Mach disc, which significantly alters the shock-cell structure. As the Mach disc forms, the large central portion of subsonic flow formed downstream of the Mach disc considerably reduces the noise generation. The data also reveals that at a high $\beta$ the turbulent mixing noise level is much lower than the underexpanded noise levels. As $\beta$ decreases, the mixing noise contribution relative to the total noise becomes increasingly significant.

Experiments by Tanna [22] and of Seiner and Norum [23] provided further insight into the characteristics of shock noise. These data include measurements from convergentdivergent (C-D) nozzles and covered a broad range of jet 


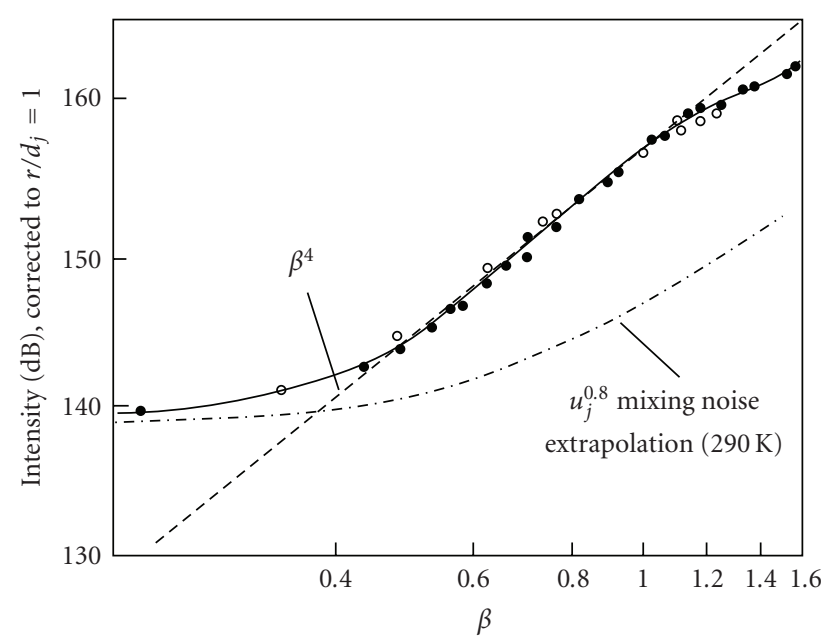

Figure 3: Intensity of broadband noise at 90 deg. to jet axis (from Fisher et al. [16]).

conditions (NPR and jet temperature ratio $T_{t} / T_{0}$ where $T_{t}$ and $T_{0}$ are, respectively, the stagnation temperature and the ambient temperature). Both Tanna's data [22], covering $\beta \leq$ $1\left(M_{j} \leq 1.41\right.$, or $\left.p_{t} / p_{0} \leq 3.5\right)$, and the data of Seiner and Norum [23] (covering the design Mach number $M_{d}=1.5$ and 2 , and $M_{j}=1$ to 2.37 or $\beta=0$ to 2.15 ) suggest trends similar to those indicated by the data of Harper-Bourne and Fisher [5] to the extent that the overall intensity of shockassociated noise is principally a function of jet pressure ratio, scales as $I \propto \beta^{4}$, and is independent of jet temperature ratio (efflux temperature) and emission angle. The data by Krothapalli et al. [18] for broadband shock noise for $M_{j}$ in the range of 1.24 to 1.66 suggest that the shock noise intensity follows the $\beta^{4}$ dependence for both the stationary ambient and in forward flight.

Directivity and spectral characteristics of BBSN were investigated experimentally by Tanna [22], Norum and Seiner [24], Pao and Seiner [25], Krothapalli et al. [18], and Jothi and Srinivasan [26]. Detailed measurements by Norum and Seiner [24] suggest that the shock noise is fairly directional at lower values of $\beta$ and approaches omnidirectionality. Test data by Tanna [22] reveal the peak frequency (which represents an important characteristic) with the angle of observation. Pao and Seiner [25] indicate that the power spectral density $(\mathrm{dB} / \mathrm{Hz})$ increases as $\omega^{4}$ below the peak frequency and decays as $\omega^{-2}$ beyond the peak frequency. Measurements by Jothi and Srinivasan [26] suggest that at higher pressure ratio exceeding about two, noncircular jets are quieter relative to circular jets by as much as $10 \mathrm{~dB}$.

\section{Studies on Shock-Turbulence Interaction}

3.1. Linear Theories. Broadly speaking, the decomposition of a general fluctuation into acoustic, vorticity, and entropy waves is well known (Kovasznay [27]). In general, any plane wave (acoustic, vorticity/shear, or entropy) interacting with a shock undergoes transformation and at the same time generates the other two waves (Zang et al. [28]). In a uniformly

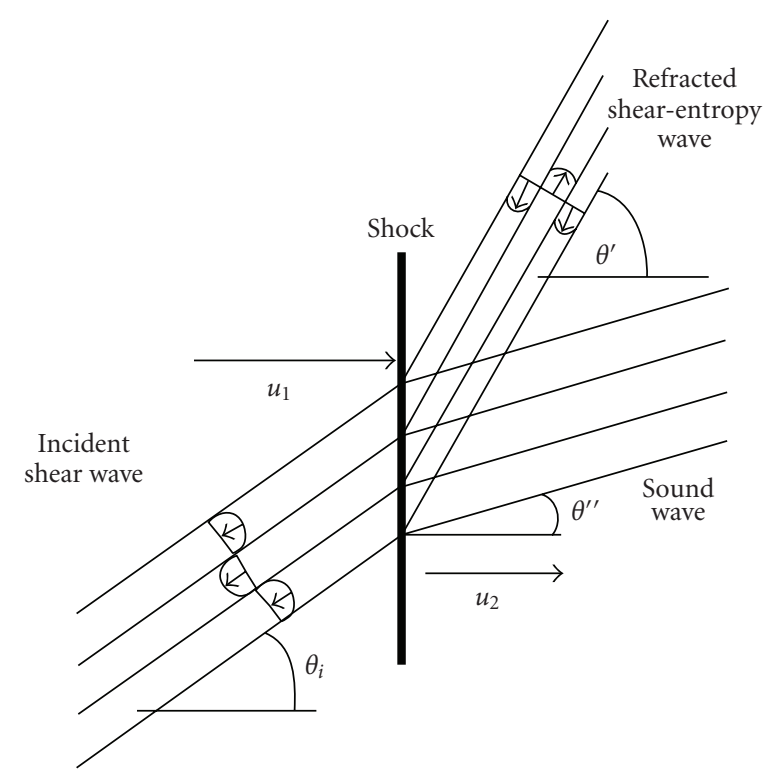

FIgure 4: Interaction of a shear wave with shock wave (Ribner [19]).

moving fluid, a general fluctuation can be decomposed into acoustic, entropy, and vorticity waves (perturbations). The acoustic waves (isentropic pressure fluctuations) propagate with the acoustic speed $c$ relative to the moving fluid, while the vorticity and the entropy waves are convected with the fluid. Linear analyses of a single wave (shear/vorticity, acoustic, or entropy) interaction with a shock wave were carried out by Blokhintzev [29], Burgers [30], Ribner [14, 15, 19, 20], Moore [31], and McKenzie and Westphal [32]. With regard to broadband shock noise, we are primarily concerned here with the generation of acoustic waves by the interaction of a shock wave with an incident vorticity wave (Figure 4) in our endeavor to investigate shock-turbulence interaction.

According to the linear theory, for sufficiently high angles of incidence for the wave ahead of the shock, the incident wave vector $\mathbf{k}$ has a nonzero imaginary part. Under such circumstances, the refracted (or generated) acoustic wave is not an infinite plane wave; instead, it exhibits an exponential decay as it propagates downstream behind the shock. The incidence angle that separates the plane wave acoustic response from the decaying ones is termed the critical angle. The critical angle is close to $90 \mathrm{deg}$. for incident acoustic waves, and roughly $60 \mathrm{deg}$. for incident vorticity and entropy waves [28]. Linear theory predicts that most transmission and generation coefficients are peaked near the critical angle. From a theoretical point of view, the actual transmission/generation coefficients are independent of the incident wavelength in the linear limit [28].

A turbulent velocity field can be represented as a superposition or spectrum of elementary waves distributed among all orientations and wavelengths in accordance with Fourier's integral theorem. The waves are transverse for weak turbulence because of the constraint of incompressibility (even though convected at high speed). Thus, a single wave can be 


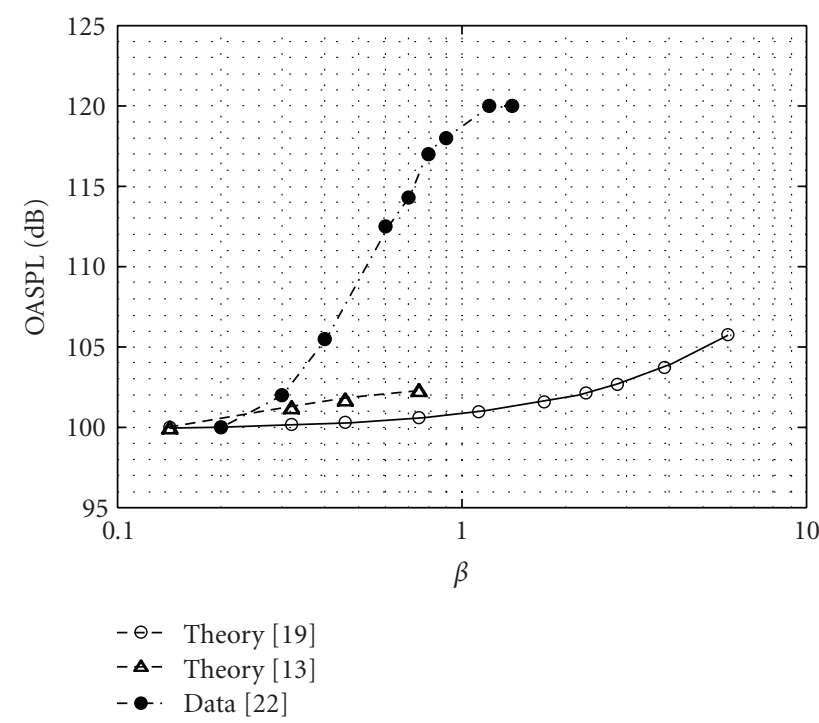

FIGURE 5: Intensity of broadband noise according to the theories of Ribner [19] and Lighthill [13].

interpreted physically as a plane sinusoidal wave of shearing motion (Batchelor [33]). According to linear interaction analysis (LIA), the vorticity waves incident at angles beyond a critical angle

$$
\theta_{c}=\theta_{c}\left(M_{1}\right)
$$

generate acoustic waves which decay as they propagate downstream. In (2), $M_{1}$ refers to Mach number upstream of the shock.

Lighthill [13] and Ribner [14, 15, 19] conducted theoretical analysis on acoustic noise generation by shock wave/turbulence interaction. In both Ribner's and Lighthill's theories, the turbulence is treated in effect as a frozen spatial pattern with neglect of temporal fluctuations.

3.1.1. Ribner's Analysis. Ribner [14] studied in detail the interaction between a vorticity wave and a shock wave. Ribner [14, 19] extended this analysis to consider a spectrum of incident vorticity waves (in three dimensions) and computed, for an isotropic incident spectrum, detailed statistics of the downstream flowfield with emphasis on the generated noise. The basic building blocks of Ribner's linear theory are oblique plane sinusoidal waves of vorticity (shear waves), see Figure 6. These represent single spectral (monochromatic) waves composed of (in 3D) an instantaneous snapshot of arbitrary flow. The waves are considered to interact independently with the shock, and then the waves are superposed to represent turbulence upstream and downstream of the shock. The detailed statistical formalism was worked out in Ribner [15] and partly summarized by Ribner [20].

The mean spectral sound pressure is expressed by [19]

$$
\overline{p^{\prime \prime 2}}=\int_{0}^{\infty}|P(\theta)|^{2}[u u] d^{3} \mathbf{k}
$$

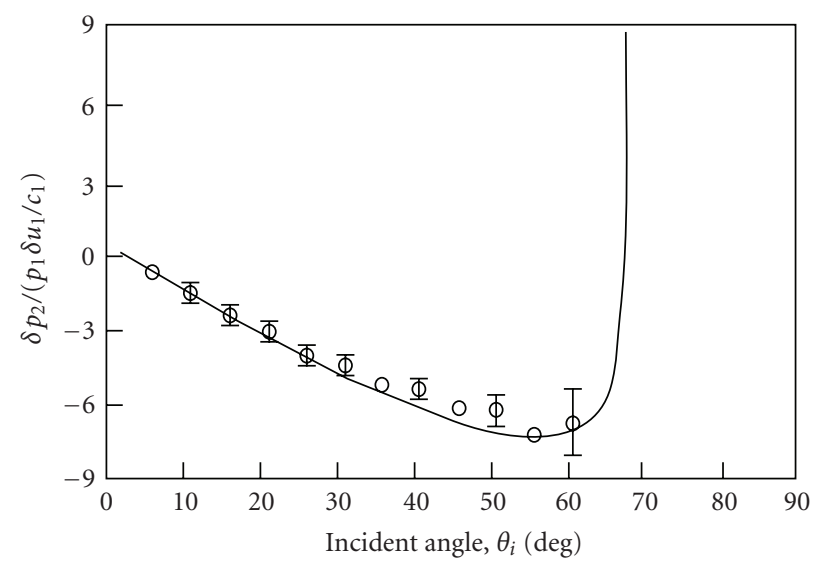

FIGURE 6: Dependence of acoustic response to vorticity waves incident on a Mach 8 shock (solid lines: linear theory, circles from nonlinear Euler simulations; from Zang et al. [28]).

where $P(\theta)$ is the transfer function for sound wave generation, and the special symbol $[u u]$ stands for the longitudinal spectral density of $\left\langle u^{2}\right\rangle$ in wave-number space $\mathbf{k}$, where $\mathbf{k}$ is a three-dimensional vector [20]. The wave number is defined by

$$
k=|\mathbf{k}|=\frac{2 \pi}{\lambda}=\frac{\omega}{c}
$$

where $\lambda$ is the acoustic wavelength and $\omega$ the circular frequency. Considering that the initial turbulence is isotropic, Ribner $[14,15]$ tabulated the transfer function $P(\theta)$ and the critical angle. Calculations of the linear theory were performed for an upstream Mach number range of $1<M_{1}<$ 10. For a one percent turbulence, the postshock noise level is predicted to exceed $140 \mathrm{~dB}$ for all preshock Mach numbers above 1.05 .

3.1.2. Lighthills's Theory. Lighthill [13] considered the generation of sound due to the interaction of turbulence with very weak shock waves (acoustic-like waves), by aid of his general theory of sound generated aerodynamically $[1,2]$. The weak shock is represented by an acoustic step function. In Lighthill's theory, the assumptions are more restrictive than in Ribner's analysis in the sense that both the shock and the turbulence are weak. As a result, the rippling motion of the shock as well as the differences in the turbulence intensity across the shock are suppressed. The ratio of freely scattered acoustic energy to the kinetic energy of turbulence traversed by the shock wave is expressed relative to a frame moving with the fluid, whereas Ribner's analysis deals with a frame attached to the shock. For a direct comparison, Ribner [19] converted the results of Lighthill [13] to the shock-fixed reference frame.

Figure 5 shows a comparison of the scattered sound intensity (in SPL) between Ribner's result [19] and that of Lighthill [13], as presented by Ribner [19]. Significant discrepancy is noted between the two results. A critical discussion of this comparison is provided by Ribner [19]. 
Note that the results of Ribner and Lighthill shown here are not to scale.

3.2. Nonlinear Euler Simulations. Since the shock weakens as the Mach number tends to unity, the shock front will undergo greater distortions from an incident wave of fixed amplitude. Thus, nonlinear effects ought to be increasingly important for lower Mach numbers (Zang et al. [28]). Zang et al. [28] validated the linear analysis of McKenzie and Westphal [32] by comparisons with their numerical solution of nonlinear 2D Euler equations. Although restricted in terms of the incident angle of the disturbance, it was shown that the linear analysis was valid over a surprisingly large range of shock strengths and disturbance amplitudes (Figure 6). In this plot, $\delta p_{2}$ represents the amplitude of the acoustic pressure generated downstream of the shock, $\delta u_{1}$ is the amplitude of the incident vorticity wave, $p_{1}$ is the mean pressure upstream of the shock, and $c_{1}$ is the sound speed upstream of the shock. The comparisons suggest that the linear theory is fairly accurate for a wide range of incident angles up to the critical angle. Although comparisons were made for both the incident and the vorticity waves, only the comparisons for the incident vorticity waves are indicated in Figure 6.

3.3. Instability Wave Theories. Tam [34] formulated a stochastic model theory of the BBSN of axisymmetric supersonic jets by considering the dynamics of weakly nonlinear interaction between the downstream propagating linear instability waves in the mixing layer and shockcell structures. On account of the solution complexity, a semiempirical (less general) shock-noise model was arrived at, valid for slightly imperfectly expanded supersonic jets. An increase in the spectral peak associated with the BBSN is attributed to the convective amplification of the sources. The theory was extended [35] to moderately imperfectly expanded jets with the aid of empirical modifications to the amplitude of the waveguide modes of the shock cell. The specific role of instability wave-shock cell interaction is discussed in the reviews [36-38] on supersonic jet noise.

3.4. DNS Simulations. The simplest circumstance in which turbulence interacts with a shock wave is the case of isotropic turbulence interacting with a normal shock (transverse vorticity amplification). Lee et al. $[39,40]$ and Mahesh et al. [41] performed DNS simulation of the interaction of 3D isotropic turbulence up to $M_{1}=3$. Detailed comparisons of DNS results to Ribner's linear analysis $[15,20]$ were made. DNS calculations $[39,40]$ and numerical simulations by Rotman [42] show that the vorticity amplification predictions are in good agreement with the linear theory. Satisfactory agreement between the DNS simulations [41] and the linear theory is noticed with regard to amplification (of turbulent kinetic energy) and anisotropy downstream of the shock (representing the ratio of longitudinal to transverse velocity fluctuation).

Although DNS solutions provide the most accurate representation of the shock/turbulence interaction, they seem to be impractical for conditions involving strong shock waves and high Reynolds number turbulence on account of resolution requirements of shock waves and turbulence.

3.5. Experimental Data. With regard to experimental data, it is found that in general compression enhances turbulence and expansion suppresses it. Measurements by Barre et al. [43] at $M_{1}=3$ suggest that the shock wave increases the longitudinal fluctuating velocity in agreement with Ribner's theory [19]. As indicated by Ribner [44], the measured amplification ratio of mean square longitudinal component of turbulence velocity $\left(u_{2}^{2} / u_{1}^{2}\right)$ is close to the theoretical value of about 1.5 as predicted by Ribner's theory $[14,15]$ at $M_{1}=$ 3. Density fluctuations in high-speed jets were investigated by Panda and Seasholtz [45].

There are also important studies dealing with the fundamental interaction between vorticity and an isolated shock. The interaction of a shock with a longitudinal vortex was treated by Erlebacher et al. [46] on the basis of analytical (linear and nonlinear) theories and numerical simulations. Grasso and Pirozzoli [47] solved 2D Euler equations with the aid of a higher-order finite volume-weighted ENO scheme in their study of sound generation in the interaction of a shock wave with a cylindrical vortex. In this connection, they also derived a Green's function for the acoustic analogy for a general vortex structure to analytically characterize the shock-vortex interaction. Direct noise computation in subsonic and supersonic jets was reviewed by Bailly et al. [48]. Avital et al. [49] investigated Mach wave radiation by mixing layers.

\section{Proposed Model}

The discrepancy between the theories of Lighthill [13] and of Ribner [19] in comparison with the experimental data for the scaling of shock noise intensity (as evident from Figure 5) requires further investigation. This discrepancy is attributed to the fact that in their theories the turbulence is treated effectively as a frozen spatial pattern without regard to the temporal fluctuations. There is thus a deficiency in applying linear theory to real turbulence, which consists of transient phenomena and not steady plane waves [28]. Also, threedimensional simulation is needed to accommodate vortex stretching [28].

The irregularity and disorderliness characterizing turbulence involve the impermanence of the various frequencies and of the various periodicities and scale (Hinze [50]). Strictly speaking, the instantaneous physical interaction process (shock/vorticity) cannot be represented by timeaveraging. In view of these circumstances, it is plausible that the peak angle of incidence is representative of the shock-shear wave interaction insofar as the scaling of the BBSN is concerned. Accordingly, it is postulated here that the shock-vorticity interaction at the peak incidence governs the generation of sound. Also, it is assumed that the interaction of turbulence with the leading shock cell forms the maximum contribution to the intensity of sound, and that the sound contribution due to the interactions at 
the subsequent shock cells is of secondary nature (subsidiary importance).

With the above postulate, the linear acoustic response (acoustic pressure rise) for shock-vortex interaction (vorticity waves incident on a shock) is computed for various upstream Mach numbers. In this context, as pointed out by Zhang et al. [28] that among the linear analyses, the work by McKenzie and Westphal [32] is more accessible and tractable than the earlier pioneering studies of Ribner $[14,15]$ and others, while yielding equivalent results. In consequence, our calculations will be based on the work of [32]. For normal shocks and an ideal gas, the relation for the acoustic response in dimensionless form becomes relatively simple and is expressed by (see [32, Equation (42)])

$$
\frac{\delta p_{2}}{\left(p_{1} / c_{1}\right) \delta u_{1}}=\frac{-4 \gamma M_{1}\left(M_{1}^{2}-1\right) \sin \theta_{1}\left[1-\left(u_{2} / u_{1}\right) \tan ^{2} \theta_{i}\right]}{(\gamma+1) D}
$$

where

$$
\begin{gathered}
D=1+M_{1}^{2}+\frac{u_{2}}{u_{1}} \tan ^{2} \theta_{i}\left(1-M_{1}^{2}\right)+2 M_{2} M_{1}^{2}\left[1-\frac{\tan ^{2} \theta_{i}}{\tan ^{2} \theta_{c}}\right]_{(4 \mathrm{~b})}^{1 / 2}, \\
\frac{M_{2}}{M_{1}}=\left[\frac{(\gamma-1) M_{1}^{2}+2}{2 \gamma M_{1}^{2}-(\gamma-1)}\right]^{1 / 2}, \\
\frac{u_{2}}{u_{1}}=\frac{\rho_{1}}{\rho_{2}}=\frac{(\gamma-1) M_{1}^{2}+2}{(\gamma+1) M_{1}^{2}}, \\
\tan ^{2} \theta_{c}=\frac{M_{1}^{2}}{R^{2}\left(1-M_{2}^{2}\right)}, \\
R^{2}=\frac{c_{2}^{2}}{c_{1}^{2}}=\frac{1+((\gamma-1) / 2) M_{1}^{2}}{1+((\gamma-1) / 2) M_{2}^{2}} .
\end{gathered}
$$

In the preceding equations, $\rho$ denotes the density, and the subscripts 1 and 2 denote the upstream and downstream of the normal shock, respectively.

On the basis of $(4 \mathrm{a})-(4 \mathrm{f})$, the acoustic response has been computed as a function of the incident angle for several upstream Mach numbers from 1.2 to 8 (Figure 7). The results point out that the peak angle of incidence and the associated acoustic response varies with the Mach number. The acoustic pressure increases with an increase in Mach number. Notice that the computed acoustic pressure at $M_{1}=8$ is identical to that shown in Figure 6, as computed by Zang et al. [28], thus verifying the present calculations. It should be pointed out that the results for various upstream Mach numbers as shown in Figure 7 are originally obtained by the author.

\section{Results}

Based on the foregoing premise, the intensity of BBSN taken at the peak incidence angle, as obtained from the results of

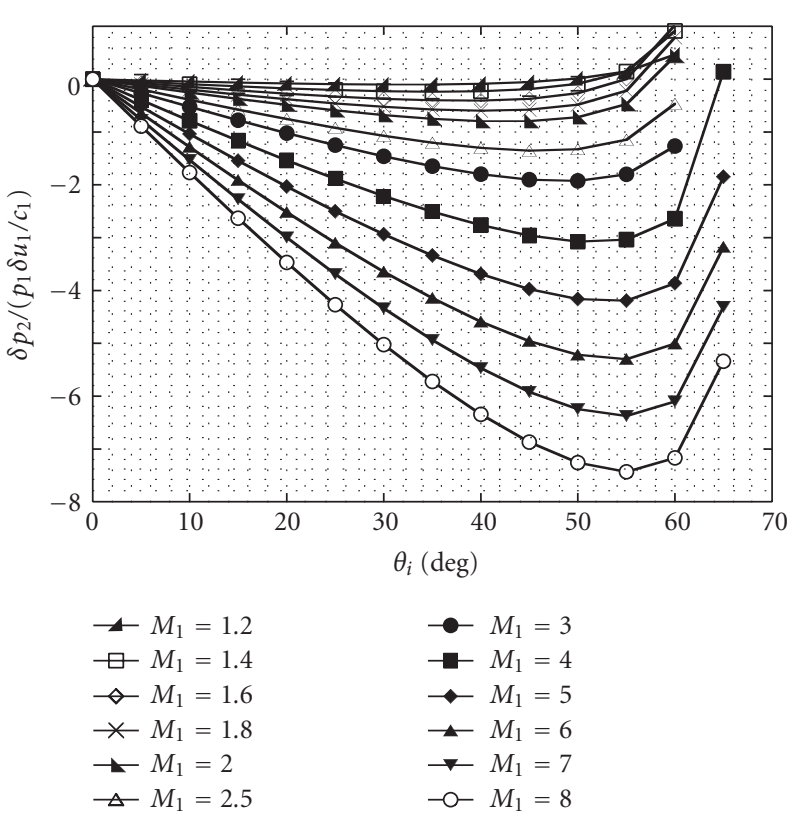

Figure 7: Pressure rise due to shock/vorticity amplification according to linear theory.

Figure 7 , is plotted as function of $\beta$ in Figure 8 . The sound pressure level (OASPL) is given by

$$
\text { OASPL }=20 \log \left(\frac{\sqrt{\left(\delta p_{2}\right)^{2}}}{p_{\text {ref }}}\right) \mathrm{dB}
$$

where $\delta p_{2}$ is obtained from (4), and $p_{\text {ref }}$ is the reference sound pressure $\left(2 \times 10^{-5} \mathrm{~N} / \mathrm{m}^{2}\right)$. It is revealed that the scaling of intensity very nearly varies as $\beta^{4}$ for a wide range of $\beta$ between 0.2 and 2.0. In this range, the present theory yields

$$
I \propto \beta^{4.2} .
$$

Beyond this range, there is seen a change in slope in the intensity variation.

A direct comparison of the scaling based on the proposed model and the experimental data of Tanna [22] is presented in Figure 9. The OASPL data are obtained from an underexpanded nozzle with $T_{t} / T_{0}=1$ (cold jet) at $\phi=135 \mathrm{deg}$. For scaling purposes, the model results presented in Figure 9 are adjusted such that at $\beta=0.7$, the prediction matches the OASPL data of $117 \mathrm{~dB}$ (taken for reference purposes). The predictions from the proposed model substantially agree with the data in the range of $0.3<\beta<1.0$. Recall that for low values of $\beta$ less than about 0.3 , the turbulent mixing noise becomes significant. It is known that beyond about $\beta=1$, a Mach disc is formed, which alters the shock-cell structure. The large central portion of subsonic flow that develops downstream of the Mach disc considerably diminishes the noise generation.

The satisfactory explanation of the $\beta^{4}$ scaling law by the proposed theory suggests that the hypothesis of peak incidence angle for the generation of sound by shock-vorticity interaction is plausible. This forms an important contribution of the present work. 


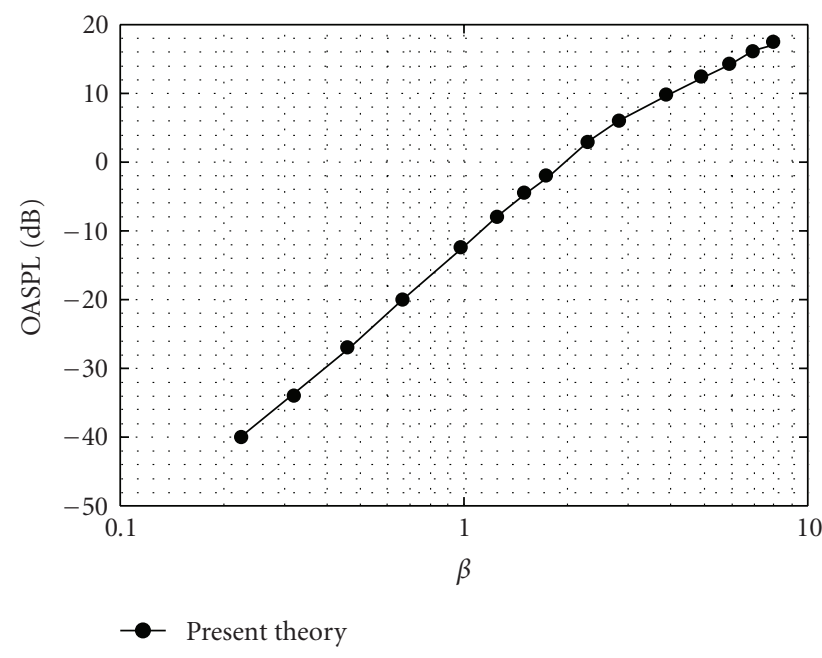

FIGURE 8: Intensity of broadband noise according to the theory of maximum incidence angle.

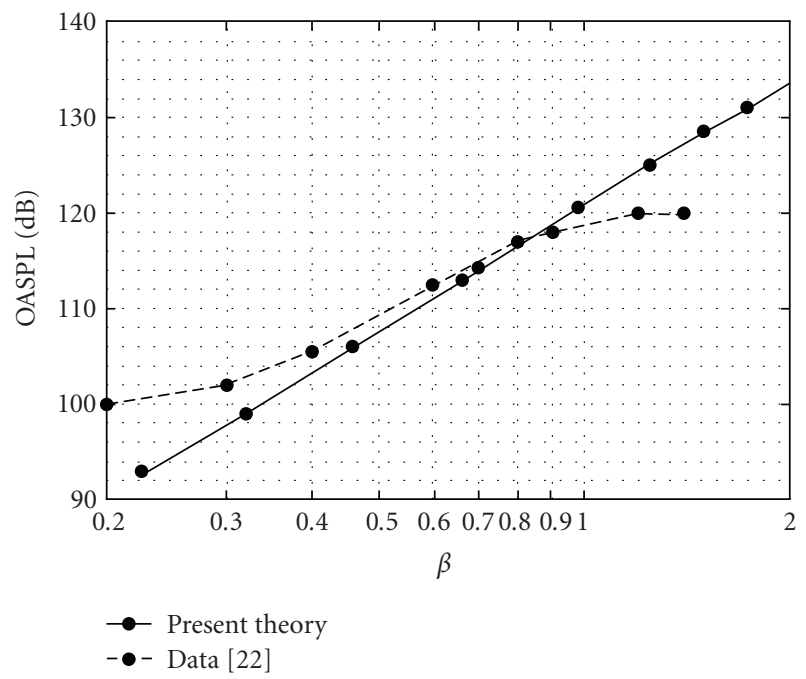

Figure 9: Comparison of the present theory and the data of Tanna [22] for the intensity of the broadband shock noise.

The determination of the directionality effects and spectral distribution of the BBSN are outside the scope of the present investigation, which is mainly concerned with the scaling law for broadband shock noise intensity. The fact that only a single shock-cell/vortex interaction is considered here indicates that the shock noise intensity obtained by the present formulation is essentially omnidirectional. It is believed that the present investigation would be helpful in our understanding of supersonic jet noise $[51,52]$ and its suppression by active control such as water injection [53-55].

\section{Discussion}

With regard to the validity of the linear theory, Kandula [7] recently applied the linear theory to the production of screech noise, regarded as a consequence of interaction of an incident acoustic wave and a shock wave, and obtained a remarkable agreement with data for the screech amplitude for fully expanded Mach number $M_{j}$ up to 2. The predicted directivity pattern is also satisfactory when compared with measurements. Since the screech amplitudes are considerably larger as relative to broadband shock noise levels, it is hardly surprising that the proposed model based on linear formulation is able to describe the intensity scaling law for broadband shock noise. As indicated in Zang et al. [28], the linear theory is found to be valid for extraordinarily large amplitudes, suggesting that the region of validity of linear theory is indeed much broader than one would generally expect. Quoting Zang et al. [28], in some of the examples of their numerical Euler simulations, the postshock velocity fluctuations were of nearly the same order as the mean stream velocity!

Referring to the notable difference, for strong shocks, between the linear approach of Ribner [19] and the weakly nonlinear approach of Lighthill [13], it may be ascribed to differences in their assumptions and simplifications in treating the statistics of the interaction process.

\section{Conclusion}

A physical basis is proposed for the scaling of the broadband shock-associated noise in supersonic jets considering linear interaction between the shock wave and the vorticity wave considering the peak incidence angle for the turbulence. The hypothesis that the generation of sound at peak incidence angle is important is shown to satisfactorily describe the experimental scaling law for the broadband shock-associated noise intensity in imperfectly expanded supersonic jets.

\section{Nomenclature}

$c$ : Speed of sound

$d_{j}$ : Nozzle diameter

$f:$ Frequency

I: Overall sound intensity, $\delta p_{2} /\left(\rho_{0} c^{2}\right)$

k: Incident wave vector

$k$ : Wave number

$M$ : Mach number

$M_{d}$ : Design Mach number

$M_{j}$ : Fully expanded jet Mach number

p: $\quad$ Pressure

$r$ : Distance to measurement location

$R: \quad c_{2} / c_{1}$

St: Strouhal number, $f d_{j} / u_{j}$

T: Temperature

$u$ : Velocity

$u_{j}$ : Jet exit velocity

$\gamma$ : Isentropic exponent

$\delta p_{2}$ : Amplitude of the acoustic pressure rise

$\delta u_{1}$ : Amplitude of the incident vorticity wave

$\phi$ : Angle from downstream jet axis

$\lambda$ : Acoustic wavelength

$\rho$ : Density 
$\theta_{c}:$ Critical angle

$\omega$ : Circular frequency, $2 \pi f$.

\section{Subscripts}

e: Nozzle exit

$t$ : Stagnation (reservoir)

0: Ambient

1: Upstream of shock

2: Downstream of shock.

\section{Acknowledgment}

The author would like to thank Dr. Eldad Avital (Reader in Computational Fluids and Acoustics School of Engineering and Materials, Queen Mary University of London) for helpful suggestions in improving the paper.

\section{References}

[1] M. J. Lighthill, "On sound generated aerodynamically-I. General theory," Proceedings of the Royal Society of London. Series A, vol. 211, no. 1107, pp. 564-587, 1952.

[2] M. J. Lighthill, "On sound generated aerodynamically-II. Turbulence as a source of sound," Proceedings of the Royal Society of London. Series A, vol. 222, no. 1148, pp. 1-32, 1954.

[3] J. E. Ffowcs-Williams, "The noise from turbulence convected at high speed," Proceedings of the Royal Society of London. Series A, vol. 255, no. 1061, pp. 469-503, 1963.

[4] J. M. Seiner, "Advances in high speed jet aeroacoustics," in Proceedings of the AIAA/NASA 9th Aeroacoustics Conference, 1984, AIAA paper 84-2275.

[5] M. Harper-Bourne and M. J. Fisher, "The noise from shock waves in supersonic jets," in Proceedings of the AGARD Conference on Noise Mechanisms, 1973, CP-131, paper 11.

[6] A. Powell, "The noise of choked jets," Journal of the Acoustical Society of America, vol. 25, no. 3, pp. 385-389, 1953.

[7] M. Kandula, "Shock-refracted acoustic wave model for screech amplitude in supersonic jets," AIAA Journal, vol. 46, no. 3, pp. 682-689, 2008.

[8] R. Emden, "Über die ausstromungserscheinungen permanenter gase," Annalen der Physik, vol. 69, pp. 264-289, 1899.

[9] L. Prandtl, "Über die stationären Wellen in einem Gasstrahl," Physik Zeitschrift, vol. 5, no. 19, pp. 599-601, 1904.

[10] J. W. S. Rayleigh, "On the discharge of gases under high pressure,” Philosophical Magazine, vol. 32, pp. 177-181, 1916.

[11] D. C. Pack, "A note on Prandtl's formula for the wavelength of a supersonic gas jet," Quarterly Journal of Mechanics and Applied Mathematics, vol. 3, pp. 173-181, 1950.

[12] A. Powell, "On Prandtl's formulas for supersonic jet cell length," International Journal of Aeroacoustics, vol. 9, no. 1-2, pp. 207-236, 2010.

[13] M. J. Lighthill, "On the energy scattered from the interaction of turbulence with sound or shock waves," Proceedings of Cambridge Philosophical Society, vol. 49, pp. 531-551, 1953.

[14] H. S. Ribner, "Convection of a pattern of vorticity through a shock wave," NACA Report, no. 1164, 1954.

[15] H. S. Ribner, "Shock-turbulence interaction and the generation of noise," NACA Report, no. 1233, 1955.
[16] M. J. Fisher, P. A. Lush, and M. Harper Bourne, "Jet noise," Journal of Sound and Vibration, vol. 28, no. 3, pp. 563-585, 1973.

[17] M. S. Howe and J. E. Ffowcs Williams, "On the noise generated by an imperfectly expanded supersonic jet," Philosophical Transactions of the Royal Society of London, vol. 289, no. 1358, pp. 71-314, 1978.

[18] A. Krothapalli, P. T. Soderman, C. S. Allen, J. A. Hayes, and S. M. Jaeger, "Flight effects on the far-field noise of a heated supersonic jet," AIAA Journal, vol. 35, no. 6, pp. 952-957, 1997.

[19] H. S. Ribner, "Acoustic energy flux from shock-turbulence interaction," Journal of Fluid Mechanics, vol. 35, no. 2, pp. 299310, 1969.

[20] H. S. Ribner, "Spectra of noise and amplified turbulence emanating from shock-turbulence interaction," AIAA Journal, vol. 25, no. 3, pp. 436-442, 1987.

[21] M. Kandula, "On the scaling law for broadband shock noise intensity in supersonic jets," AIAA-2009-3318, 2009.

[22] H. K. Tanna, "An experimental study of jet noise-part II. Shock associated noise," Journal of Sound and Vibration, vol. 50, no. 3, pp. 429-444, 1977.

[23] J. M. Seiner and T. D. Norum, "Experiments of shockassociated noise in supersonic jets," AIAA-79-1526, 1979.

[24] T. D. Norum and J. M. Seiner, "Broadband shock noise from supersonic jets,” AIAA Journal, vol. 20, no. 1, pp. 68-73, 1982.

[25] S. P. Pao and J. M. Seiner, "Shock-associated noise in supersonic jets," AIAA Journal, vol. 21, no. 5, pp. 687-693, 1983.

[26] T. J. S. Jothi and K. Srinivasan, "Acoustic characteristics of non-circular slot jets," Acta Acustica United with Acustica, vol. 94, no. 2, pp. 229-242, 2008.

[27] L. S. G. Kovasznay, “Turbulence in supersonic flow," Journal of Aerospace Sciences, vol. 20, no. 10, pp. 657-674, 1953.

[28] T. A. Zang, M. Y. Hussaini, and D. M. Bushnell, "Numerical computation of turbulence amplification in shock-wave interactions," AIAA Journal, vol. 22, no. 1, pp. 13-21, 1984.

[29] D. I. Blokhintzev, "A moving sound pickup," Doklady Akademii Nauk SSSR, vol. 47, pp. 22-25, 1945, translation: Acoustics of a nonhomogeneous moving medium, NACA TM 1399, 1956.

[30] J. M. Burgers, "On the transmission of sound waves through a shock wave," Proceedings of the Koniklijke Nederlandse van Wetenshappaen, vol. 49, pp. 273-281, 1946.

[31] F. K. Moore, "Unsteady oblique interaction of a shock wave with a plane disturbance," NACA 1165, 1954.

[32] J. F. Mckenzie and K. O. Westphal, "Interaction of linear waves with oblique shock waves," Physics of Fluids, vol. 11, no. 11, pp. 2350-2362, 1968.

[33] G. K. Batchelor, The Theory of Homogeneous Turbulence, Cambridge University Press, Cambridge, UK, 1953.

[34] C. K. W. Tam, "Stochastic model theory of broadband shock associated noise from supersonic jets," Journal of Sound and Vibration, vol. 116, no. 2, pp. 265-302, 1987.

[35] C. K. W. Tam, "Broadband shock-associated noise of moderately imperfectly expanded supersonic jets," Journal of Sound and Vibration, vol. 140, no. 1, pp. 55-71, 1990.

[36] C. K. W. Tam, "Supersonic jet noise," Annual Reviews of Fluid Mechanics, vol. 27, pp. 17-43, 1995.

[37] S. K. Lele, "Phased array models of shock-cell noise sources," AIAA-2005-2841, 2005.

[38] G. Raman, "Advances in supersonic jet screech: review and perspective," Progress in Aerospace Sciences, vol. 34, no. 1-2, pp. 45-106, 1998. 
[39] S. Lee, S. K. Lele, and P. Moin, "Direct numerical simulation of isotropic turbulence interacting with a weak shock wave," Journal of Fluid Mechanics, vol. 251, pp. 533-562, 1993.

[40] S. Lee, S. K. Lele, and P. Moin, "Interaction of isotropic turbulence with shock waves: effect of shock strength," Journal of Fluid Mechanics, vol. 340, pp. 225-247, 1997.

[41] K. Mahesh, S. K. Lele, and P. Moin, “The influence of entropy fluctuations on the interaction of turbulence with a shock wave," Journal of Fluid Mechanics, vol. 334, pp. 353-379, 1997.

[42] D. Rotman, "Shock wave effects on a turbulent flow," Physics of Fluids A, vol. 3, no. 7, pp. 1792-1806, 1991.

[43] S. Barre, D. Alem, and J. P. Bonnet, "Experimental study of a normal shock/homogeneous turbulence interaction," AIAA Journal, vol. 34, no. 5, pp. 968-974, 1996.

[44] H. S. Ribner, "Comment on experimental study of a normal shock/homogeneous turbulence interaction," AIAA Journal, vol. 36, no. 2, p. 494, 1998.

[45] J. Panda and R. G. Seasholtz, "Experimental investigation of density fluctuations in high-speed jets and correlation with generated noise," Journal of Fluid Mechanics, vol. 450, pp. 97130, 2002.

[46] G. Erlebacher, M. Y. Hussaini, and C. W. Shu, "Interaction of a shock with a longitudinal vortex," Journal of Fluid Mechanics, vol. 337, pp. 129-153, 1997.

[47] F. Grasso and S. Pirozzoli, "Shock-wave-vortex interactions: shock and vortex deformations, and sound production," Theoretical and Computational Fluid Dynamics, vol. 13, no. 6, pp. 421-456, 2000.

[48] C. Bailly, C. Bogey, and O. Marsden, "Progress in direct noise computation," International Journal of Aeroacoustics, vol. 9, no. 1-2, pp. 123-143, 2010.

[49] E. J. Avital, N. D. Sandham, and K. H. Lou, "Mach wave radiation by mixing layers-part I: analysis of the sound field," Theoretical and Computational Fluid Dynamics, vol. 12, no. 2, pp. 73-90, 1998.

[50] J. O. Hinze, Turbulence, McGraw-Hill, New York, NY, USA, 2nd edition, 1975.

[51] M. Kandula, "On the scaling laws and similarity spectra for jet noise in subsonic and supersonic flow," International Journal of Acoustics and Vibrations, vol. 13, no. 1, pp. 3-16, 2008.

[52] M. Kandula, "Near-field acoustics of clustered rocket engines," Journal of Sound and Vibration, vol. 309, no. 3-5, pp. 852-857, 2008.

[53] M. Kandula, "Prediction of turbulent jet mixing noise reduction by water injection," AIAA Journal, vol. 46, no. 11, pp. 2714-2722, 2008.

[54] M. Kandula, "Broadband shock noise reduction in turbulent jets by water injection," Applied Acoustics, vol. 70, no. 7, pp. 1009-1014, 2009.

[55] M. Kandula, "Spectral attenuation of sound in dilute suspensions with nonlinear particle relaxation," Journal of the Acoustical Society of America, vol. 124, no. 5, pp. EL284-EL290, 2008. 

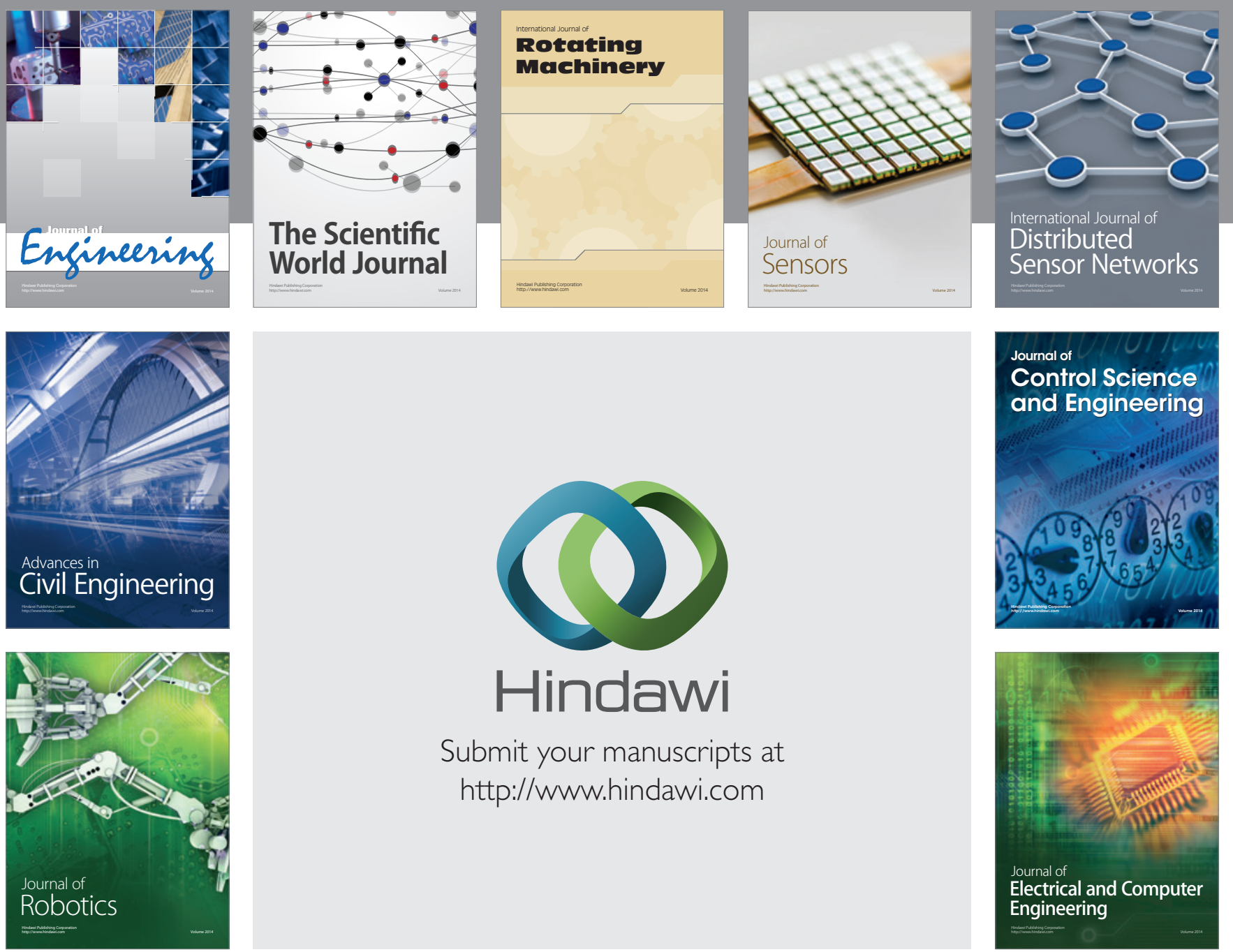

Submit your manuscripts at

http://www.hindawi.com
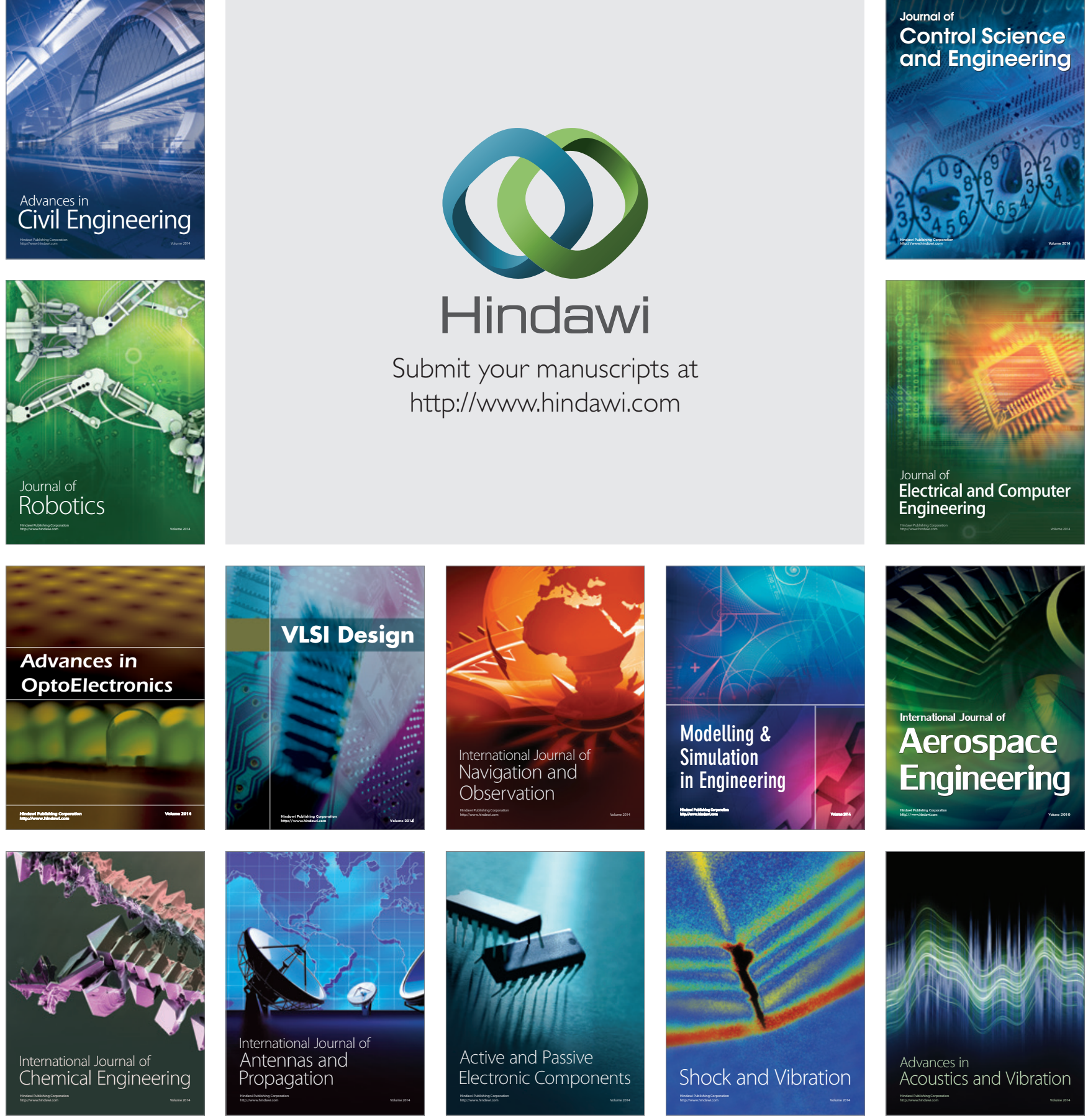